DOI:10.30842/ielcp230690152313

Grigory Vorobyev

(Институт лингвистических исследований РАН, Санкт-Петербург)

\title{
EINIGE SPERLINGSVÖGEL UND EINE SCHLANGE. NOCH EINMAL ZU ARIST. HIST. AN. 592B22
}

Автор предлагает два дополнения к своей недавней статье, в

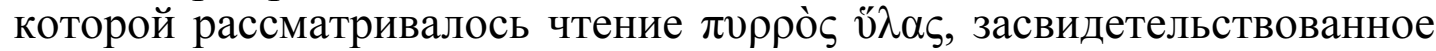
в ряде рукописей «Истории животных» Аристотеля вместо общепри-

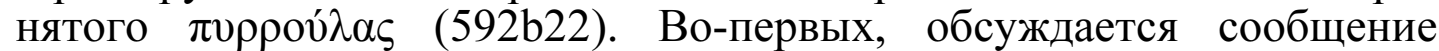
Д’Арси Томпсона (1895 г.) об употреблении лиррои́ $\lambda \alpha \varsigma$ в новогреческом для обозначения снегиря. Выдвигается предположение об источнике этого утверждения и делается вывод о его нерелевантности для отождествления предполагаемого аристотелевского названия птицы

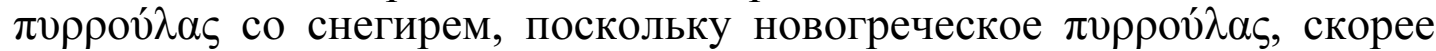
всего, восходит не к устной традиции, а к ученой литературе, опирающейся на Аристотеля. Следовательно, отождествление $\pi \nu \rho \rho o v ́ \lambda \alpha \varsigma$ со снегирем предстает менее вероятным и появляется

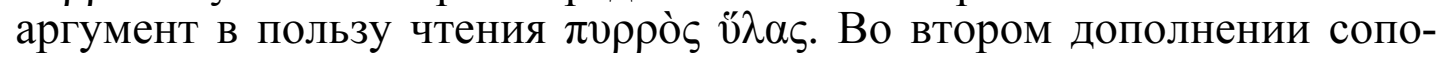

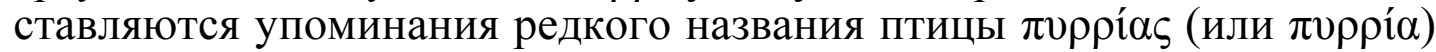
и омонимичного названия змеи в Ath. 2, 69, 3, Dionys. Per. Ixeut. 3, 13, 22 и Hsch. 4461. В результате в Ath. 2, 69, 3 вместо конъектуры Клавдия Салмазия предлагается другая.

Ключевые слова: Аристотель, Афиней, Дионисий Периегет, Гесихий Александрийский, снегири, зарянки, зоологическая номенклатура, названия птиц в древнегреческом, названия птиц в новогреческом

Grigory Vorobyev

(Institute for Linguistic Studies, RAS, St. Petersburg)

\section{Some Passeriformes and a snake. Again on Arist. Hist. An. 592b22}

The article presents two addenda to the author's recent study concerning the manuscript variants $\pi v \rho \rho o v ́ \lambda \alpha \varsigma$ and $\pi v \rho \rho o ̀ \varsigma ~ v ँ \lambda \alpha \varsigma$ in Arist. Hist. An. 592b22. In that previous work, an attempt was made to trace back the Latin fortune of the Greek vidas. Now, we scrutinize D'Arcy W. Thompson's assertion $\pi v \rho \rho o v ́ \lambda \alpha \varsigma$ means 'bullfinch' in Modern Greek. Thompson mistakenly refers to Theodor von Heldreich - it is apparently Demetrios Bikelas who he is quoting. The latter, in turn, could have taken

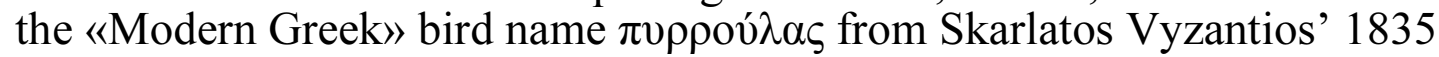
dictionary. Given Vyzantios' purist and prescriptive approach to lexicography, he must have drawn the word from a learned source based on Aristotle rather than from a vernacular one close to the oral tradition. That is why Thompson's «Modern Greek» argumentation for identifying

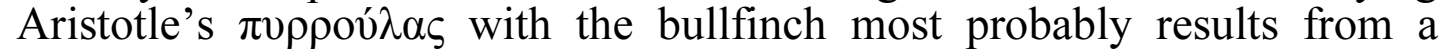
vicious circle. This corroborates Carl Jacob Sundevall's identification of $\pi v \rho \rho o v i \lambda \alpha \varsigma$ with the robin and, furthermore, increases the plausibility of the 


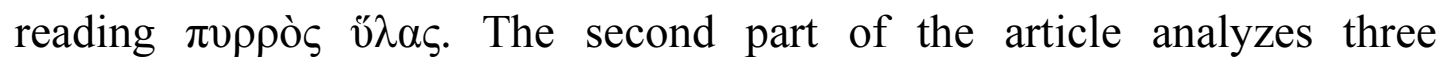
testimonies of the rare bird name $\pi v \rho \rho i \alpha \varsigma / \pi v \rho \rho i ́ \alpha$ and of the homonymous denomination of a snake. Although apparently irrelevant for assessing the variant readings in Arist. Hist. An. 592b22, these words deserve examination. Namely, a comparison of manuscript readings and possible emendations in Ath. 2, 69, 3, Dionys. Per. Ixeut. 3, 13, 22 and Hsch. 4461 suggests that Claudius Salmasius' conjecture in Ath. 2, 69, 3 should be rejected. Another conjecture is ventured instead.

Keywords: Aristotle, Athenaeus, Dionysius Periegetes, Hesychius of Alexandria, bullfinches, robins, zoological nomenclature, bird names in Ancient Greek, bird names in Modern Greek

Der vorliegende Artikel bietet zwei Nachträge zu unserem früheren Aufsatz (Vorobyev 2018).

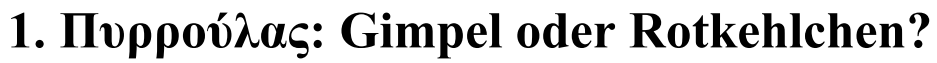

Im genannten Beitrag wurde gezeigt, dass der neulateinische Vogelname sylvia im 15. Jahrhundert als Übersetzung des Wortes vँ $\lambda \alpha \varsigma$ entstand, das seinerseits als Teil des Wortpaars $\pi v \rho \rho o ̀ \varsigma ~ v ̋ \lambda \alpha \varsigma$ (Arist. Hist. An. 592b22) belegt ist. Die seit Aldus Manutius von allen Editoren in den Text aufgenommene Variante lautet aber $\pi v \rho \rho o v ́ \lambda \alpha \varsigma$ (Manutius 1497: f. 107r), mit einer willkürlichen Verschiebung des Akzents. Die handschriftlich belegten Lesarten sind

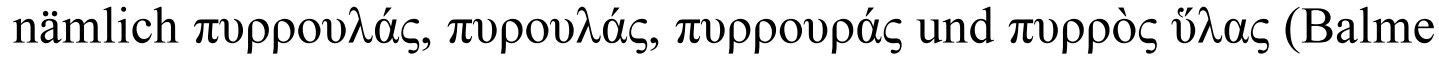
2002: 342). Die Wörterbücher behalten den Akzent von Aldus bei.

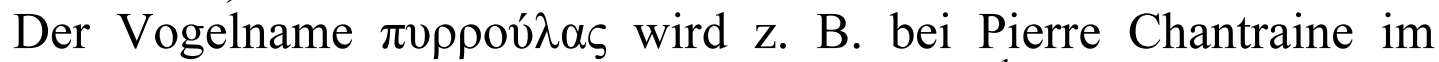
Artikel über das Adjektiv $\pi v \rho \rho o ́ \varsigma ~ ' f e u e r f a r b e n$ ' folgendermaßen erläutert: das Substantiv sei mithilfe des "suffixe diminutif et expressif» -ovג- von ebendiesem Adjektiv abgeleitet (Chantraine 1999: $959)^{2}$.

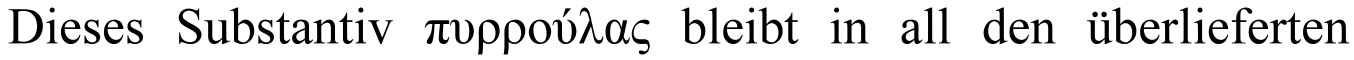
Schreibweisen ein Hapax legomenon. Es ist also kein Wunder, dass es nur sehr schwierig mit einer bekannten Vogelart identifiziert werden kann. Das einzige nämlich, was Aristoteles über diesen Vogel berichtet, ist seine Zugehörigkeit zu den Würmer- bzw.

${ }^{1} \pi v \rho \rho o ́ \varsigma$ 'feuerfarben, feuerrot, rötlich, in verschiedenen Abstufungen der Farben, bis zum Blonden hin' (Pape 1880: 825); 'flame-coloured, yellowish-red; with red hair; tawny; red; blushing' (Liddell, Scott, Jones 1940: s. v.); 'rougeâtre, roux' (Chantraine 1999: 959). — Ich bedanke mich ganz herzlich bei Hannes Amberger für das sorgfältige Korrekturlesen und bei Maxim Kisilier für seine Hinweise.

${ }^{2}$ In den Wörterbüchern von Hjalmar Frisk und Robert Beekes erscheint das

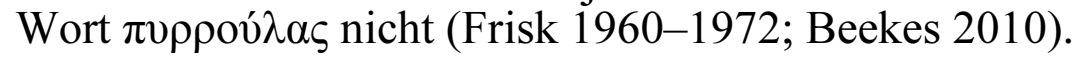


Insektenlarvenfressern $(\sigma \kappa \omega \lambda \eta \kappa o \varphi \alpha ́ \gamma \alpha)^{3}$. Trotz dieses Informations-

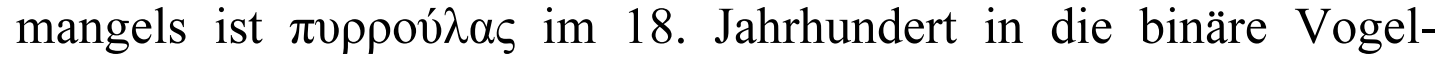
nomenklatur gelangt und wird immer noch als Name der Gattung und der Art Gimpel benutzt: Pyrrhula (Brisson, 1760) und Pyrrhula pyrrhula (Linnaeus, 1758) ${ }^{4}$. Im 19. Jahrhundert wurde allerdings

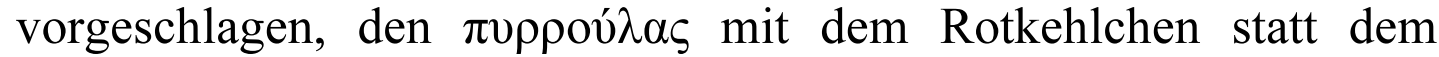
Gimpel gleichzusetzen. Im Folgenden wird in diesem Sinne gegen die Identifikation mit dem Gimpel argumentiert.

Der englische Naturforscher William Turner hat in seinem 1544 veröffentlichten Buch als erster versucht, zu den antiken Vogelnamen systematisch zeitgenössische volkssprachliche Entsprechungen zu finden. Er hat das Wort $\pi v \rho \rho o v ́ \lambda \alpha \varsigma$, das er in dieser Form offensichtlich einer gedruckten Aristoteles-Edition entnommen hatte, als Gimpel/Dompfaff/Blutfink identifiziert, und zwar nur aufgrund der Übereinstimmung zwischen der Farbe des Gimpels und der vermutlichen Etymologie des griechischen Wortes ${ }^{5}$. Seitdem galt diese Identifikation als die richtige und wurde im 18. Jahrhundert in die binäre Nomenklatur aufgenommen.

Drei Jahrhunderte nach Turner hat der schwedische Zoologe Carl Jacob Sundevall in seinem bis heute aktuellen Werk «Die Tierarten des Aristoteles» vorgeschlagen, den $\pi v \rho \rho o v ́ \lambda \alpha \varsigma$ mit dem Rotkehlchen gleichzusetzen, denn der Gimpel ernähre sich nicht von Würmern bzw. Insektenlarven, sondern von Körnern, während das Rotkehlchen sowohl der von Aristoteles beschriebenen Ernährungsweise des $\pi v \rho \rho o v ́ \lambda \alpha \varsigma$, als auch seiner aufgrund der Etymologie vermuteten Gefiederfarbe entspreche. Darüber hinaus hat Sundevall

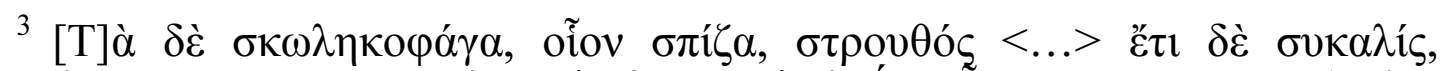

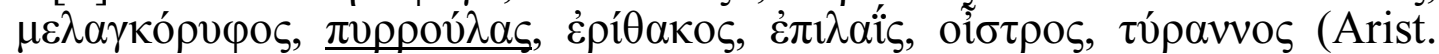
Hist. an. 592b16-23), zitiert nach der Edition Balme 2002: 342.

${ }^{4}$ Zur Systematik und Nomenklatur der Gimpel s.: Collar, Newton, Bonan 2018; Bezzel 1993: 600-679 (zur ganzen Familie Fringillidae), sowie Boehme, Flint 1994: 436 (speziell zur Gattung Pyrrhula).

${ }^{5}$ «Ego, nominis etymologiam secutus, rubicillam Anglorum bulfincam et Germanorum bloudvincam esse conjicio» («Meinerseits vermute ich, der Etymologie des Namens folgend, dass rubicilla der bullfinch der Engländer und der Blutfink der Deutschen ist», Turner 1544: f. I1v-I2r, im Kapitel

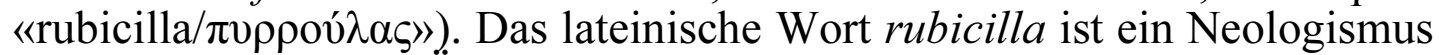
aus Theodor Gazas Übersetzung von Arist. Hist. An. 592b22, das für

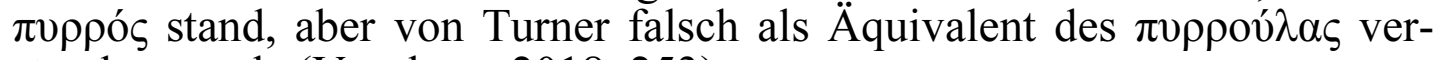
standen wurde (Vorobyev 2018: 253). 
bemerkt, dass die Gimpel lediglich im nördlichen Griechenland und fast nur im Gebirge zu sehen sind (Sundevall 1863: 111) ${ }^{6}$.

Trotzdem behauptete D'Arcy W. Thompson in seinem 1895 erschienenen «Glossary of Greek birds», dass unter $\pi v \rho \rho o v ́ \lambda \alpha \varsigma$ doch der Gimpel zu verstehen sei, denn laut Theodor von Heldreichs «La faune de Grèce» bezeichne dasselbe Wort im Neugriechischen eben den Gimpel ${ }^{7}$. Anscheinend hat hier Thompson Heldreichs Werk irrtümlich zitiert, denn Heldreich versteht unter $\pi \nu \rho \rho o v ́ \lambda \alpha \varsigma$ ganz im Gegenteil das Rotkehlchen, indem er Sundevalls Identifikation übernimmt (Heldreich 1878: 41). Über die neugriechischen Bezeichnungen für den Gimpel berichtet Heldreich gar nichts und identifiziert ihn, allerdings mit Fragezeichen, mit dem altgriechischen ebenfalls

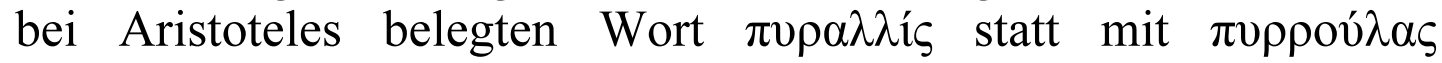
(Heldreich 1878: 44).

Vielleicht bezog sich Thompson in der Tat nicht auf Heldreich, sondern auf den kurz nach Heldreichs Werk veröffentlichten und von Thompson auch sonst benutzten Beitrag von Demetrios Bikelas (Vikelas), der Heldreichs Liste der Tiere Griechenlands wesentlich erweitert hat. Bikelas erwähnt tatsächlich unter den seit der Antike fast nicht modifizierten bis ganz unverändert gebliebenen Vogelnamen das neugriechische Wort $\pi v \rho o u ́ \lambda \alpha \varsigma$ (sic). Er übersetzt es als 'Gimpel' 8 und führt als altgriechisches Äquivalent das Wort $\pi v \rho \alpha \lambda \lambda i$ is an (Bikélas 1878: 215). Diese Identifikation mit $\pi v \rho \alpha \lambda \lambda i \varsigma$ beruht offensichtlich auf der Vermutung Heldreichs. Es bleibt aber unklar, ob Bikelas' Gleichsetzung des Gimpels und des neugriechischen $\pi$ voov́ $\lambda \alpha_{\varsigma}$ sich auf die neugriechische Alltags- oder, im Gegenteil, Wissenschaftssprache bezieht, wobei die letztere gewiss von der lateinischen Nomenklatur und von Aristoteles-Editionen abhängig war. Falls das von Bikelas erwähnte Wort $\pi v \rho o v ́ \lambda \alpha \varsigma$ aus solchen gelehrten Quellen in den demotischen Wortschatz gelangt

${ }^{6}$ Sundevall bezieht sich auf den wissenschaftlichen Namen des Rotkehlchens Luscinia rubecula (mit dem Druckfehler «Lucinia»). Heute wird Erithacus rubecula als gültiger wissenschaftlicher Name benutzt.

${ }^{7}$ «Sundevall $<\ldots>$ identifies $\pi$ uppovi $\lambda \alpha \varsigma$ with the Robin, the Bullfinch being a seed-eater, and confined to the mountainous parts of Northern Greece: but Heldreich quotes the same word as the name for the Bullfinch in $\operatorname{Mod}<\mathrm{ern}>\mathrm{G}<\mathrm{ree}>\mathrm{k} \gg$ (Thompson 1895: 152). Obwohl Heldreichs Werk in den bei Thompson 1895: 205 angegebenen bibliographischen Abkürzungen fehlt, lässt es sich anhand der (wenn auch selten präsenten) Seitenangaben schließen, dass der schottische Gelehrte eben auf Heldreichs 1878 veröffentlichte «Faune de Grèce» verweist.

${ }^{8}$ Er nennt ihn auf Latein Pyrrhula vulgaris, was eine frühere wissenschaftliche Bezeichnung des jetzt gültigen Namens Pyrrhula pyrrhula ist. 
war, kann seine Verwendung im Neugriechischen des 19. Jahrhundert die Richtigkeit der Identifikation des aristotelischen $\pi \nu \rho \rho o v ́ \lambda \alpha \varsigma$ mit dem Gimpel auf keinen Fall beweisen. Wir hätten es dann mit einem Circulus vitiosus zu tun. Versuchen wir also den Ursprung des bei Bikélas 1878 belegten neugriechischen $\pi v \rho o v ́ \lambda \alpha \varsigma$ mithilfe der lexikographischen Quellen zu klären.

1835 erschien das nach dem Thesaurus von Gerasimos Vlachos (Vlachos 1659) erste von einem Griechen in Griechenland veröffentlichte Wörterbuch der griechischen Volkssprache, nämlich das Lexikon des Skarlatos Vyzantios (Stathi 2006: 147; Mackridge 2009: 166-167; Lavidas 2018: 64). Vyzantios bietet in seinem Wörterbuch, in dem zeitgenössische demotische Wörter ins Altgriechische und ins Französische übersetzt werden, den Eintrag $\pi v \rho o v ́ \lambda \alpha \varsigma$ (mit der Variante $\pi v \rho \rho o v ́ \lambda \alpha \varsigma$ ). Der Artikel enthält aber nichts außer dem Verweis auf einen anderen Eintrag, nämlich $\chi \varepsilon 1 \mu \omega v i \alpha ́ \tau \eta \varsigma$ (Vyzantios 1835: 243). Für das neugriechische Lemma $\chi \varepsilon 1 \mu \omega v i \alpha ́ \tau \eta \varsigma$ schlägt Vyzantios zwei Bedeutungen vor. Erstens versteht er darunter die altgriechischen Wörter $\pi \hat{v} \rho \rho \alpha$ und $\pi v \rho \alpha \lambda \lambda i \varsigma^{9}$. Er betrachtet sie als Synonyme und führt kein französisches Äquivalent an. Zweitens erklärt er das Wort $\chi \varepsilon 1 \mu \omega v i \alpha ́ \tau \eta \zeta$ als altgriechisches $\pi$ voov́ $\lambda \alpha \varsigma$, unter Angabe von vier französischen Übersetzungen, die alle Namen des Gimpels sind: pivoine, bouvreuil usw. (Vyzantios 1835: 312) ${ }^{10}$. Für Vyzantios heißt es also seltsamerweise: neugr. $\pi v(\rho) \rho o v ́ \lambda \alpha \varsigma=$ neugr. $\chi \varepsilon 1 \mu \omega v i \alpha ́ \tau \eta \varsigma ;$ neugr. $\chi \varepsilon 1 \mu \omega v i \alpha ́ \tau \eta \varsigma$ (I) $=$ altgr. $\pi \hat{v} \rho \rho \alpha / \pi v \rho \alpha \lambda \lambda i ́ s ;$ neugr. $\chi \varepsilon 1 \mu \omega v i \alpha ́ \tau \eta s$ (II) $=$ altgr. $\pi v(\rho) \rho o v ́ \lambda \alpha \varsigma=$ Gimpel.

Auch das moderne zwölfbändige Wörterbuch von Dimitrios Dimitrakos, das sowohl die alt-, als auch die mittel- und neugriechische Phase der Sprachentwicklung mit einbezieht, enthält den Eintrag $\pi \nu \rho \rho o v ́ \lambda \alpha \varsigma^{11}$. Dimitrakos fügt als «neuere, demotische» Schreibung die Variante $\pi$ $\rho_{\rho} \rho 0 v \lambda \alpha \varsigma$ bei und definiert das Wort als

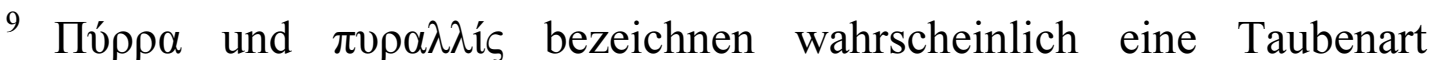
(Vorobyev 2018: 250 Anm. 12-13).

to Vyzantios bemerkt, dass es im Französischen noch weitere 19 Bezeichnungen für den Gimpel gibt. Danach verweist er mit einem «siehe

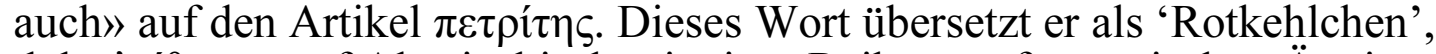

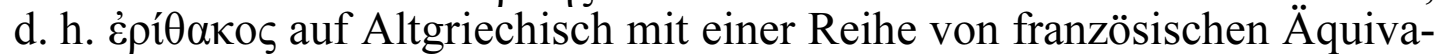
lenten, die mit rouge-gorge anfängt (Vyzantios 1835: 224; in einer er-

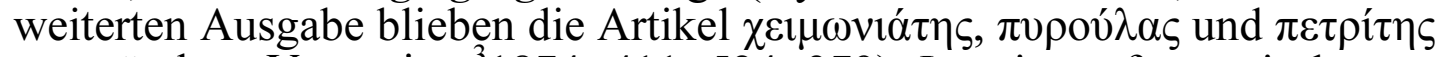
unverändert: Vyzantios ${ }^{3} 1874:$ : 411, 524, 379). In seinem französisch-neugriechischen Wörterbuch übersetzt Vyzantios das französische bouvreuil

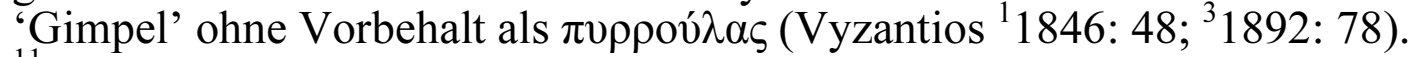

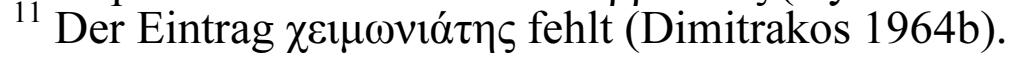


«feuerfarbige Vogelart, vielleicht der Singvogel Gemeiner Gimpel

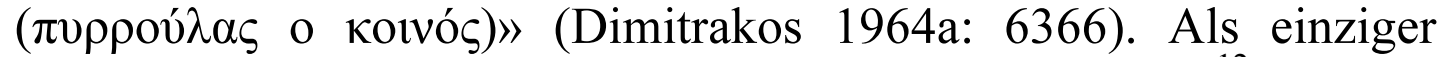
Beleg wird unsere Stelle Arist. Hist. an. 592b22 angeführt ${ }^{12}$.

Die Tatsache, dass Vyzantios und Dimitrakos das Wort $\pi v \rho o v ́ \lambda \alpha \varsigma / \pi v \rho \rho o v ́ \lambda \alpha \varsigma / \pi v ́ \rho \rho o v \lambda \alpha \varsigma$ als ein demotisches betrachten, zumal mit Doppelkonsonanten- und Betonungsschwankungen, könnte zwar bestätigen, dass es seit der Antike im ununterbrochenen Usus gewesen war. Falls also der Volksmund im Norden Griechenlands, wo der Gimpel vorkommt ${ }^{13}$, diesen Vogel unabhängig von der westlichen wissenschaftlichen Nomenklatur und von dem

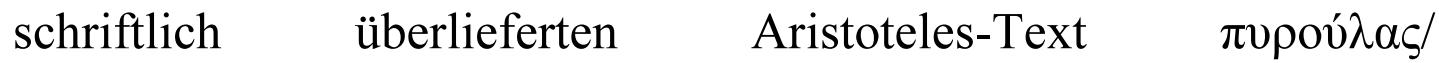
$\pi$ Argument gegen Sundevall gültig ist und das Wort bei Arist. Hist.

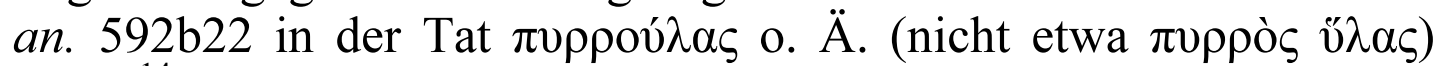
lauten $^{14}$ und wahrscheinlich eben den Gimpel bezeichnen musste.

Dabei ist aber erstaunlich, dass anscheinend keine Wörterbücher des Neugriechischen außer Vyzantios 1835 und Dimitrakos 1964 $\pi v \rho \rho o v ́ \lambda \alpha \varsigma$ erwähnen ${ }^{15}$. In der Tat, da das Wort $\pi v \rho o v ́ \lambda \alpha \varsigma$ für

12 «

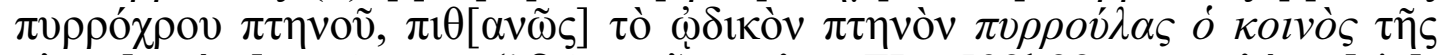

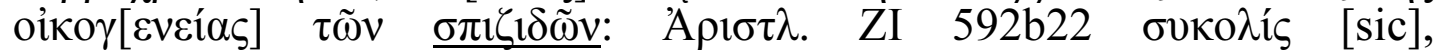

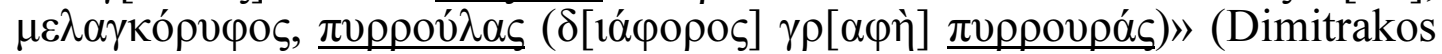
1964a: 6366).

13 Vgl. in der Datenbank «Filotis» eine Karte der griechischen Verbreitungsgebiete des Gimpels (Zustand für das Jahr 1994): http://filotis.itia.ntua.gr/species/d/6060/ (letzter Zugriff am 8.2.2019).

${ }^{14}$ D. h. Aldus Manutius hatte eine korrekte editorische Entscheidung getroffen (s. oben und Vorobyev 2018: 249).

${ }^{15}$ In den anderen Wörterbüchern der mittel- und neugriechischen Sprache, die wir durchsucht haben, gibt es Einträge weder zu $\pi v \rho(\rho)$ ov́ $\lambda \alpha \varsigma /$

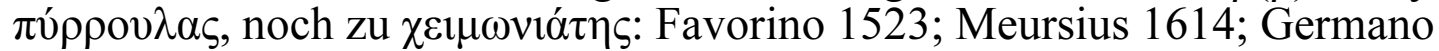
1622; Porzio 1635; Vlachos 1659; Du Cange 1668; Sommevoire 1709; Koumanoudis 1900; Sophocles ${ }^{1} 1870,{ }^{2} 1900$; Trapp et al. 2001; Lampe 1961; Andriotis ${ }^{2} 1967$ (die späteren Auflagen sind uns unzugänglich geblieben); Triantafillidis 1998; Babiniotis 2004; Babiniotis 2010; Kriaras 2012 (für das Letztere ist allerdings der Band für den Buchstaben $\chi$ noch nicht erschienen). Leider konnten wir die Wörterbücher Proïas 1935, Stamatakos 1949-1955 und Kriaras 1995 nicht konsultieren. Was die Dialektwörterbücher angeht, haben wir nur in Liapis 1996 (Pelion) und Papadopoulos 1961 (Pontos) nachschlagen können: dort sind die in Frage kommenden Vogelnamen nicht zu finden. Die (sowieso geographisch nicht zutreffenden) Wörterbücher des zypriotischen Dialekts enthalten zwar den Eintrag $\pi v ́ \rho 0 v \lambda \lambda \circ \varsigma$, er ist aber sicher irrelevant, denn dieses Wort bedeutet

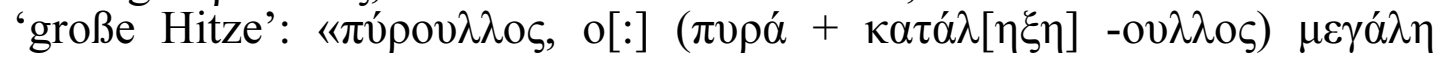

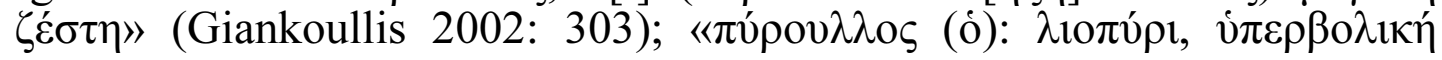


Vyzantios, wie wir gesehen haben, auch ein altgriechisches Äquivalent des neugriechischen $\chi \varepsilon \mu \omega v i \alpha ́ \tau \eta \zeta$ ist, und gleichzeitig als neugriechisches Lemma einen selbständigen Wörterbucheintrag bildet, konnte er sich wohl auf irgendeine neugriechische bildungssprachliche Quelle stützen, die ihrerseits das Wort $\pi$ vovó $\lambda$ as einer Aristoteles-Ausgabe oder einer westlichen naturwissenschaftlichen Quelle entnommen hatte. Dass das Wort $\pi v \rho \rho o v ́ \lambda \alpha \varsigma$ für Vyzantios unklar und nicht wirklich zeitgenössisch ist, wird auch dadurch bestätigt, dass er in seinem anderen, altgriechischen Wörterbuch dasselbe Wort mithilfe zweier moderner (кoıvoí) Übersetzungen

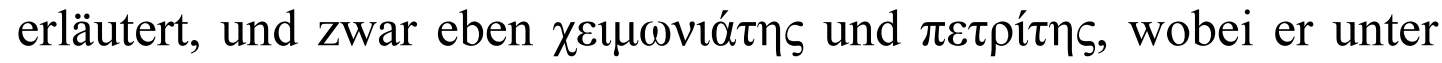
dem letzteren Vogelnamen, wie oben gezeigt, das Rotkehlchen verstand (Vyzantios ${ }^{1} 1839$ : 1109; $\left.{ }^{3} 1895: 1227\right)^{16}$. Vor allem muss berücksichtigt werden, dass Vyzantios' Ansatz puristisch und präskriptiv war (Lavidas 2018: 64-65), deshalb wäre es erstaunlich, wenn er einen raren Vogelnamen aus einem nordgriechischen Dialekt (und nicht aus Aristoteles oder einer bildungssprachlichen Quelle) in sein Wörterbuch aufgenommen hätte. Man bemerke auch, wie Dimitrakos noch im Jahr 1964 unsicher ist, welchen Vogel das Wort $\pi v \rho \rho o v ́ \lambda \alpha \varsigma$ bezeichnet, und ihn nur unter Vorbehalt $(\langle\pi \imath \theta \alpha v \tilde{\omega} \varsigma »)$ als den Gimpel identifiziert.

Wegen des griechischen Sprachenstreits war Vyzantios $1835 \mathrm{im}$ 19. Jahrhundert das letzte Wörterbuch der griechischen Volkssprache $^{17}$. Es ist also durchaus wahrscheinlich, dass der oben zitierte Artikel Bikélas 1878 als Belegstelle für die Angabe des «neu-

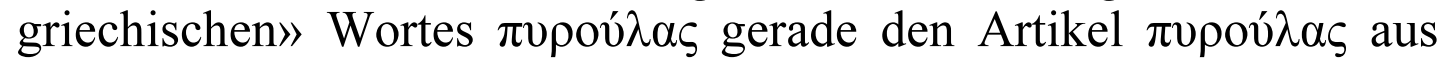
Vyzantios' Wörterbuch benutzt hat. Obwohl Bikélas zu $\pi v \rho o v ́ \lambda \alpha \varsigma$ keine Erläuterungen bietet, beruft er sich sonst häufig in Anmerkungen zu anderen Tiernamen auf Vyzantios 1835, d. h. er verwendete weitgehend dieses Wörterbuch. Wenn Thompson also

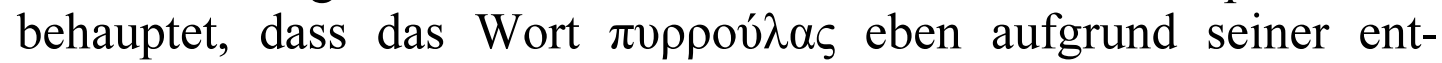
sprechenden Verwendung im Neugriechischen 'Gimpel' habe bedeuten müssen, zieht er fast sicher einen Zirkelschluss. Der Grund für diesen Zirkelschluss scheint die Tatsache, dass die damals

$\pi v \rho \alpha ́ / \zeta c ́ \delta \tau \eta, ~ \kappa \alpha ́ \psi \alpha, ~ \kappa \alpha u ́ \sigma \omega v \alpha \varsigma$ s. m. dead-heat, desert-heat, hamsin, excessively hot weather» (Papangelou 2001: 1014).

${ }^{16}$ Zur Geschichte der mittel- und neugriechischen Lexikographie s. Stathi 2006; Georgakas, Georgakas 1990; Lavidas 2018.

17 «For the next 100 years, only bilingual dictionaries were compiled, and even these covered only the Katharevousa vocabulary. An exception was Stephanos Koumanoudis's dictionary of neologisms» (Stathi 2006: 147). Stathi meint hier Koumanoudis 1900. 
zugänglichen neugriechischen Quellen (wohl Vyzantios' Wörterbuch) nicht wirklich den demotischen Usus widerspiegelten ${ }^{18}$.

Die Möglichkeit, das Wort $\pi v \rho \rho o v ́ \lambda \alpha \varsigma$ mit einer bestimmten Vogelart sicher zu identifizieren, wurde schon im 18. Jh. bezweifelt. Albert-Gaston Camus, der Autor der ersten französischen Übersetzung der aristotelischen «Tiergeschichte», hat zwar, der seit William Turner existierenden Tradition folgend, das Wort als bouvreuil 'Gimpel' wiedergegeben (Camus 1783: T. 1, 469; T. 2, 26-27), gestand aber: «J'avoue que c'est un peu au hazard que j'ai employé le nom de Bouvreuil. <..> J'ai été entraîné par le sentiment commun des Auteurs. Je crains cependant quelque erreur» (Camus 1783: T. 2, 141) ${ }^{19}$.

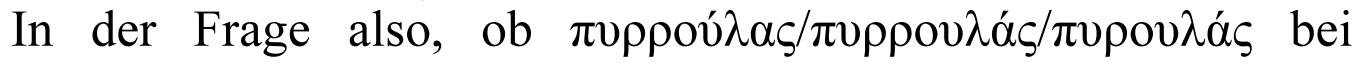
Aristoteles den Gimpel, das Rotkehlchen, einen anderen Vogel oder

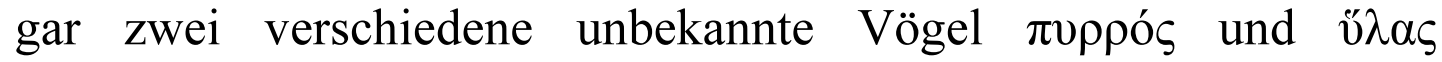
bezeichnete, ist Thompsons neugriechisches Argument für die Deutung als 'Gimpel' höchstwahrscheinlich ungültig. Sundevalls Argument über die Ernährung erscheint plausibler. Deswegen ist

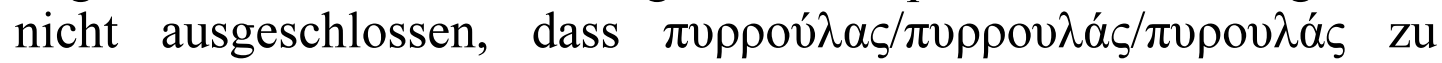
Aristoteles' Zeiten in der Tat für 'Rotkehlchen' stand. In den textkritischen Auseinandersetzungen wird nun zudem die Lesart $\pi v \rho \rho o ̀ \varsigma ~ v ̋ \lambda \alpha \varsigma$ auch nicht mehr unmöglich erscheinen.

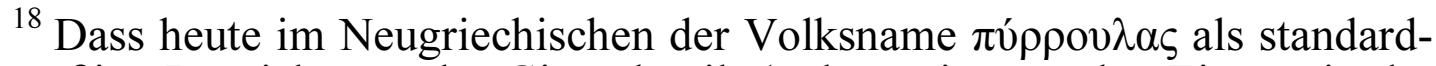
mäßige Bezeichnung des Gimpels gilt (vgl. wenigstens den Eintrag in der Database Filotis, Anm. 13 oben), muss wohl durch denselben Einfluss der gelehrten Tradition erklärt werden, wobei die lokalen Namen wahrscheinlich verschieden sind, etwa der von Vyzantios erwähnte

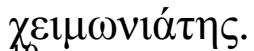

${ }^{19}$ Die deutschen Übersetzer des Traktats, Hermann Aubert und Friedrich Wimmer, transliterieren in ihrem Text vorsichtig das griechische Wort (Aubert, Wimmer 1868: Bd. 2, 131; vgl. ibid., Bd. 1, 107). Dies ist auch die Lösung David M. Balmes in seiner englischen Übersetzung (Balme 1991: 101). Jules Tricot in der neueren französischen Übersetzung folgt dem Beispiel seines Vorgängers Camus: er bezeichnet zwar in einer Fußnote die Identifikation mit dem Gimpel als zweifelhaft, verwendet jedoch im Text das Wort bouvreuil 'Gimpel' (Tricot 1957: 511 Anm. 5); in der Übersetzung von Pierre Louis erscheint bouvreuil ohne Anmerkungen (Louis 1969: 15). Auch die in den frühen 1940er Jahren entstandene russische Übersetzung von Vladimir Karpov bietet für $\pi v \rho \rho o v ́ \lambda \alpha \varsigma$ das Äquivalent снегирь 'Gimpel' (Karpov 1996: 310). Die italienischen Übersetzer meinen, dass keiner der in Arist. Hist. an. 592b21-23 aufgezählten Vogelnamen identifizierbar sei, wagen trotzdem das Wort $\pi v \rho \rho o v ́ \lambda \alpha \varsigma$ als einziges davon zu übersetzen, und zwar als ciuffolotto 'Gimpel' (Lanza, Vegetti 1971: 435 und Anm. 46). 


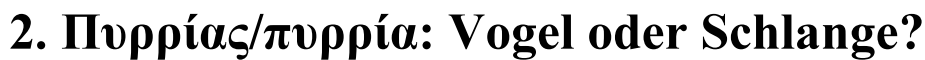

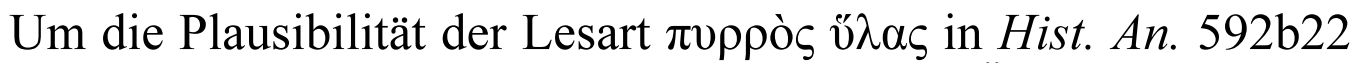
$\mathrm{zu}$ bewerten, haben wir in Vorobyev 2018 die Überlieferung der Vogelnamen $\pi v \rho \alpha \lambda$ í und $\pi v \rho \rho \alpha$ behandelt. Ein weiteres ähnliches Wort, $\pi v \rho \rho i ́ \alpha \varsigma$, scheint zwar für die Besprechung von $\pi v \rho \rho o ̀ \varsigma ~ v ̋ \lambda \alpha \varsigma$ irrelevant zu sein, denn in der handschriftlichen Überlieferung von

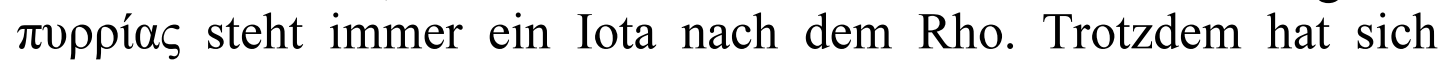

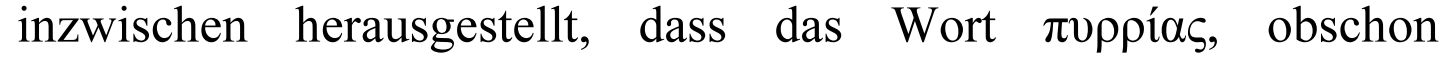
unabhängig von Arist. Hist. An. 592b22, einer Auseinandersetzung wert ist.

Der Eintrag $\pi v \rho \rho i ́ \alpha \varsigma$ in Thompsons «Glossary of Greek birds» (Thompson 1895: 152 u. 52) stützt sich nur auf eine einzige Belegstelle, nämlich auf Alexander von Myndos ap. Ath. 2, 69, 3.

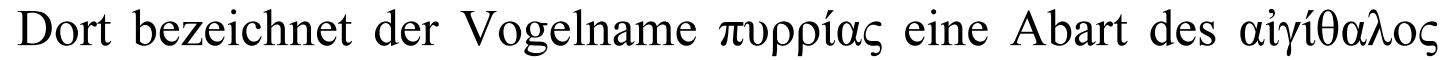

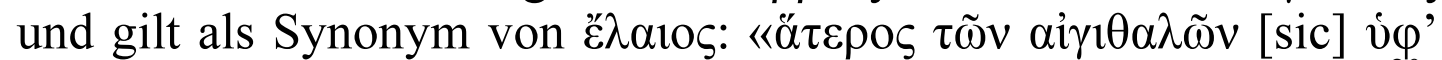

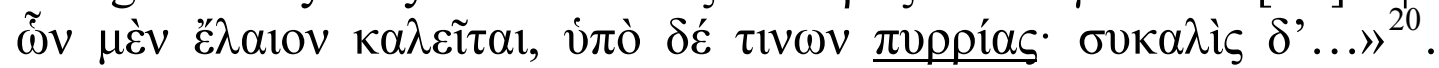
Nach Georg Kaibels Edition von Athenaios lautet die hier handschriftlich überlieferte Lesart $\pi \imath \imath^{\prime} \alpha$ bzw. $\pi \imath \iota \iota$. Gemäß Kaibel ist dabei in einem Kodex vermerkt, dass diese Lesart fehlerhaft sei. In den Text übernimmt Kaibel darum eine andere Variante, $\pi \nu \rho \rho i ́ \alpha \varsigma$, die nichts anderes ist als eine von Claudius Salmasius (1588-1653) vorgeschlagene Emendation (Kaibel 1887: 152). Salmasius schlug

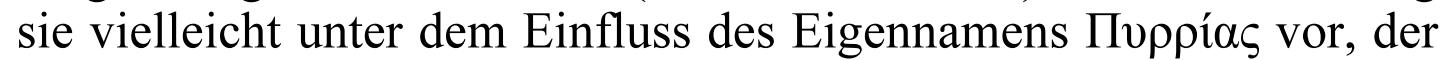
als verbreiteter Name für rothaarige Sklaven gilt (vgl. in Ar. Ran. 730).

Um die Richtigkeit der Konjektur von Salmasius beurteilen zu können, ziehen wir nun zwei weitere Belegstellen in Betracht, die bisher anscheinend noch nie in diesem Zusammenhang behandelt worden sind.

Für Salmasius' Konjektur spricht der folgende Beleg. Das Lexikon von Hesychius enthält den Eintrag $\pi v \rho \rho i ́ \alpha$, , allerdings nicht als Bezeichnung für einen Vogel, sondern für eine Schlange:

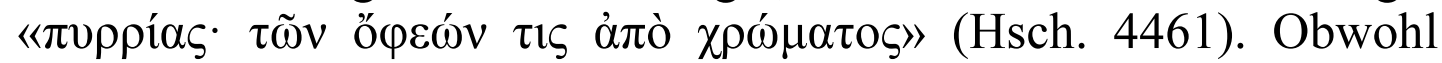
(zum Teil) feuerfarbene Schlangen im Mittelmeerraum wirklich vorkommen ${ }^{21}$, erscheint allerdings nicht ausgeschlossen, dass

\footnotetext{
${ }^{20}$ «Der eine der Aigithalen wird von einigen Elaios genannt, von anderen Pyrrias. Die Sykalis...» Sykalis ist dabei die zweite Abart des $\alpha i \gamma i \theta \alpha \lambda$ os.

${ }^{21} \mathrm{Vgl}$. wenigstens die seit dem Pleistozän belegte und im Mittelmeerraum weit verbreitete Leopardnatter, Zamenis situla (Linnaeus, 1758) (s. Wallach, Williams, Boundy 2014: 810). Es ist jedoch zu bemerken, dass sich der Schlangenname $\pi u \rho \rho i ́ a s$ nicht unbedingt, wie Hesychius ver-
} 
Hesychius hier nicht eine Schlange, sondern doch einen Vogel meinte. Ein Kopist hätte nämlich in seiner Vorlage ỏ $\rho v \varepsilon ́ \omega v$ mit ö $\varepsilon \omega v$ verwechseln können ${ }^{22}$. Unter dieser Voraussetzung wäre Salmasius' Konjektur bei Athenaios stimmig.

Eine weitere Quelle scheint aber gegen Salmasius' Vorschlag zu sprechen. In der Prosa-Epitome des Gedichts über den Vogelfang

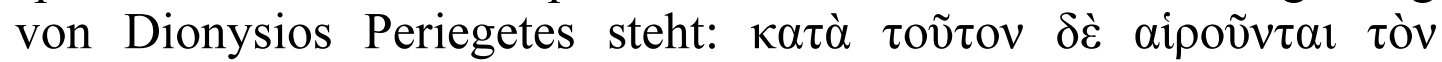

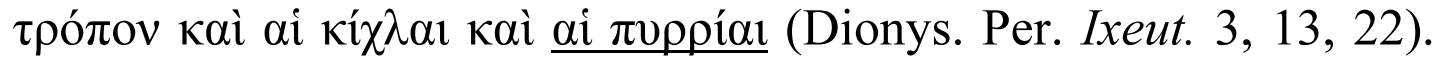
Gemäß der Edition von Antonio Garzya lesen hier die Handschriften

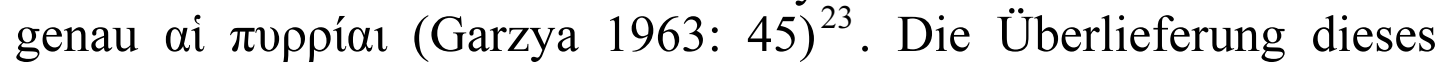
Textes deutet also daraufhin, dass der Vogelname tatsächlich $\dot{\eta} \pi v \rho \rho i ́ \alpha$ lauten sollte; deswegen müsste wohl bei Athenaios die Lesart der Kodizes $\pi \imath \rho i ́ \alpha / \pi \imath \rho \imath \alpha$ als $\pi v \rho \rho i ́ \alpha$ emendiert werden statt als

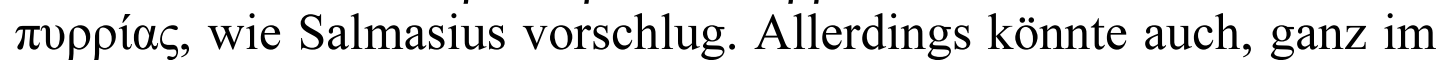
Gegenteil, ai $\pi v \rho \rho i ́ \alpha$ bei Dionysios Periegetes zu oi $\pi v \rho \rho i ́ \alpha$ emendiert werden. Die folgende Tabelle stellt die drei Textstellen möglichen Konjekturen gegenüber.

\begin{tabular}{|c|c|c|}
\hline Textstelle & $\begin{array}{l}\text { Überlieferte } \\
\text { Lesart }\end{array}$ & Rekonstruierbares Lexem \\
\hline $\begin{array}{l}\text { A. } \\
\text { Ath. } 2,69,3\end{array}$ & $\pi \imath \rho i ́ \alpha$ & $\begin{array}{l}-\pi v \rho \rho i ́ \alpha \text { (diese Konjektur wird hier } \\
\text { vorgeschlagen) } \\
-\pi v \rho \rho i ́ \alpha \varsigma \text { (Salmasius' Konjektur) } \\
\text { In beiden Fällen ist eine Emendation } \\
\text { notwendig }\end{array}$ \\
\hline $\begin{array}{l}\text { B. } \\
\text { Dionys. Per. } \\
\text { Ixeut. } 3,13,22 \\
\end{array}$ & 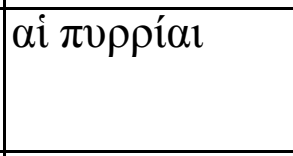 & $\begin{array}{l}-\pi v \rho \rho i ́ \alpha \text { (keine Emendation notwendig) } \\
-\pi v \rho \rho i ́ \alpha \varsigma \text { (der Artikel muss zu oi } \\
\text { emendiert werden) }\end{array}$ \\
\hline $\begin{array}{l}\text { C. } \\
\text { Hsch. } 4461\end{array}$ & 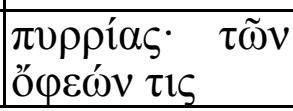 & 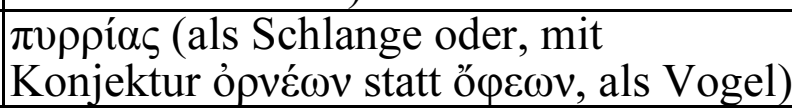 \\
\hline
\end{tabular}

mutete, auf die Farbe, sondern auch möglicherweise - im Fall einer giftigen Schlange - auf das nach einem Biss entstandene brennende Gefühl beziehen könnte.

${ }^{22}$ In der Edition von Peter Allan Hansen werden dazu keine Konjekturen angeboten (Hansen 2005: 220).

${ }_{23} \mathrm{~S}$. zur handschriftlichen Überlieferung den neuen Aufsatz von Morgane Cariou (Cariou 2017). In der 1702 erschienenen editio princeps von

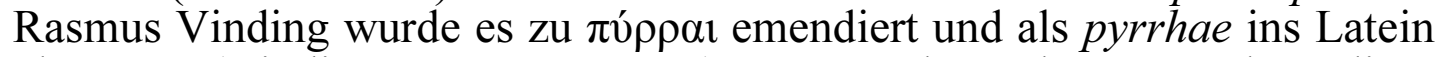
übersetzt (Vinding 1702: 102-103); Garzya bemerkt zwar, dass diese Konjektur richtig sein könnte, übernimmt sie aber nicht. 
Die vorhandenen Widersprüche können anscheinend auf drei Weisen gelöst werden:

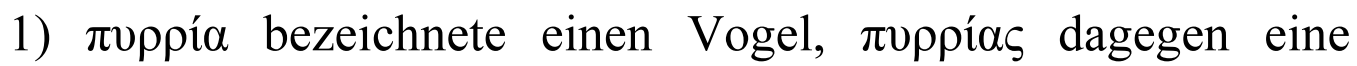
Schlange (das erfordert eine ganz einfache Emendation im Text A: von $\pi i \rho i ́ \alpha$ zu $\pi v \rho \rho i ́ \alpha)$;

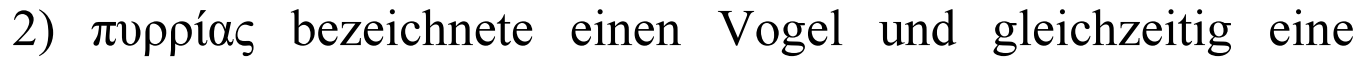
Schlange (dafür sind Emendationen in den Texten A und $\mathrm{B}$ notwendig);

3) nur der Vogelname $\pi v \rho \rho i ́ \alpha \varsigma$ existierte; kein ähnlicher Schlangenname war in Gebrauch (dafür sind Emendationen in den Texten A, B und $\mathrm{C}$ notwendig).

Die von uns im Text A vorgeschlagene Konjektur ( $\pi v \rho \rho i ́ \alpha$ statt $\pi \imath$ í $\left.^{\alpha}\right)$ erscheint phonetisch plausibler als die von Salmasius ( $\pi$ uppías statt $\pi i \rho i ́ \alpha$ ), denn es handelt sich dann nur um die Vereinfachung eines Doppelkonsonanten und um einen ItazismusFehler des Kopisten, während Salmasius' Konjektur darüber hinaus das Auslassen eines Sigma voraussetzt.

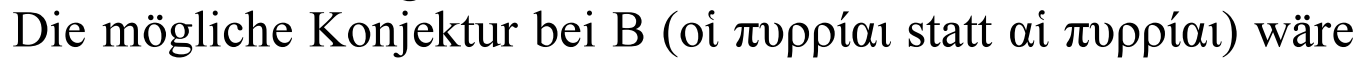
dadurch gerechtfertigt, dass der Kopist beim Abschreiben von einer Minuskelvorlage den Lektürefehler $\alpha \mathrm{l}>$ or könnte begangen haben.

Die Konjektur bei C ist paläographisch etwas schwieriger (eher die Ligatur v $\rho$ hätte mit einem Phi verwechselt werden können als das Buchstabenpaar $\rho v$ ).

Obwohl Lösung 3 eleganter erscheint, insofern sie an den drei Textstellen dasselbe Wort zu lesen vorschlägt, ist die Lösung 1 deshalb wohl plausibler, weil sie nur eine einzige, zumal ganz übliche, Konjektur voraussetzt. Lösung 1 wird auch dadurch bestätigt, dass das Wort $\pi$ $\rho \rho i ́ \alpha$ in neugriechischen Dialekten Kalabriens immer noch belegt ist, und zwar offensichtlich unabhängig von der gelehrten Tradition (Karanastasis 1991: 320, mit einemVolkslied).

\section{Literatur}

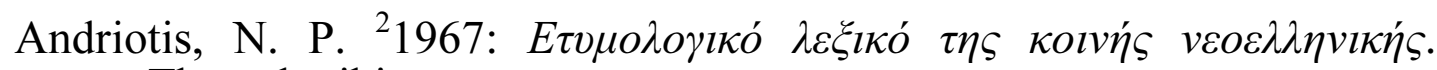
Thessaloniki.

Aubert, H., Wimmer, F. 1868.: Aristoteles Thierkunde. Kritischberichtigter Text, mit deutscher Übersetzung, sachlicher und sprachlicher Erklärung und vollständigem Index von Aubert, H., und Wimmer, F. Bd. 1-2. Leipzig.

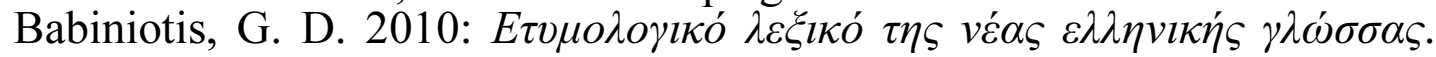
Athen.

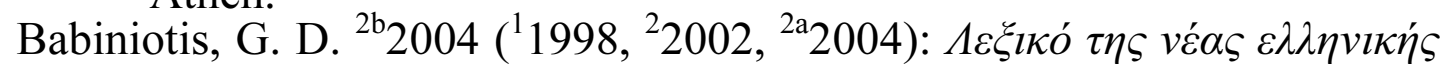
$\lambda \lambda \omega \sigma \sigma \alpha \varsigma$. Athen. 
Balme, D. M. 1991: Aristotle. History of Animals. Vol. 3: Books 7-10. Ed. and transl. by D. M. Balme. London.

Balme, D. M. 2002: Aristotle. Historia animalium. Vol. 1: Books 1-10: Text. Prepared for publication by A. Gotthelf. Cambridge.

Beekes, R. S. P. 2010: Etymological dictionary of Greek. Vols. 1-2. Leiden; Boston.

Bezzel, E. 1993: Kompendium der Vögel Mitteleuropas. Passeres. Singvögel. Wiesbaden.

Bikélas, D. 1878: Sur la nomenclature moderne de la faune grecque. Annuaire de l'Association pour l'encouragement des études grecques en France 12. 208-237.

Boehme, R. L., Flint, V. E. 1994: Pyatiiazychnyi slovar' nazvanii zhivotnykh. Ptitsy, Latinskii-russkii-angliiskii-nemetskii-frantsuzskii [A five-language dictionary of animal names. Birds. Latin, Russian, English, German, French]. Moskau.

Camus, A.-G. 1783: [Histoire des animaux d'Aristote]. T. 1. Histoire des animaux d'Aristote avec la traduction française. T. 2. Notes sur l'histoire des animaux d'Aristote. Paris.

Cariou, M. 2017: Un nouveau manuscrit de la paraphrase aux Ixeutiques de Denys dans les papiers de Conrad Gessner. Avec une note sur le «scribe de Bruxelles». Scriptorium 71, 239-268.

Chantraine, P. ${ }^{2}$ 1999: Dictionnaire étymologique de la langue grecque. Histoire des mots. Paris.

Collar, N., Newton, I., Bonan, A. 2018: Finches (Fringillidae). In: Del Hoyo, J., Elliott, A., Sargatal, J., Christie, D. A., Juana, E. de. Handbook of the birds of the world alive. Barcelona (https://www.hbw.com/ node/52376, letzter Zugriff am 8.2.2019).

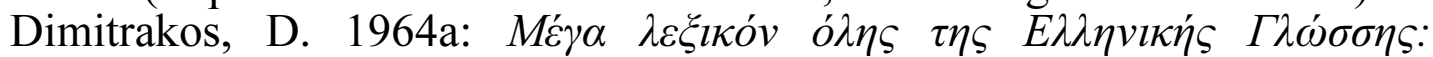

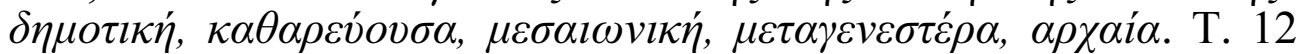

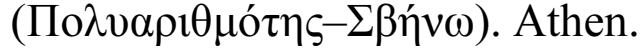

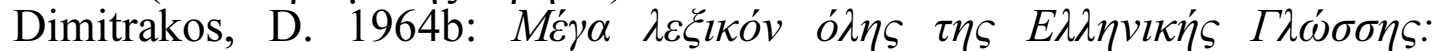

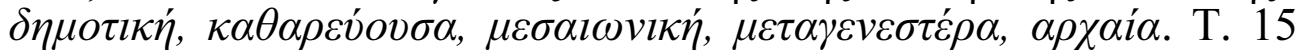
(Y

Du Cange, Ch. 1668: Glossarium ad scriptores mediae et infimae Graecitatis. T. 1-2. Lyon.

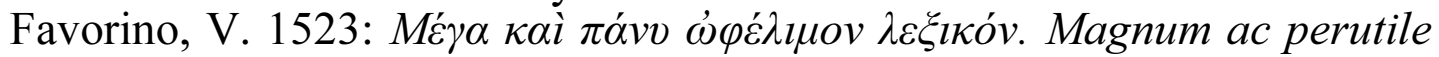
dictionarium. Rom.

Frisk, Hj. 1960-1972: Griechisches etymologisches Wörterbuch. Bd. 1-3. Heidelberg.

Garzya, A. 1963: Dionysii Ixeuticon seu De aucupio libri tres in epitomen metro solutam redacti. Leipzig.

Georgakas, D. J., Georgakas, B. 1990: The lexicography of Byzantine and Modern Greek. In: Hausmann, F. J., Reichmann, O., Wiegand, H. E., Zgusta, L. Wörterbücher. Dictionaries. Dictionnaire. Ein internationales Handbuch zur Lexikographie. An international encyclopedia of lexicography. Encyclopédie internationale de lexicographie. Vol. 2. Berlin; New York. 1705-1713.

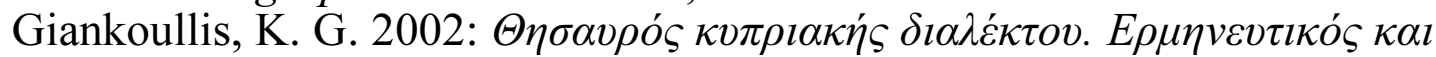

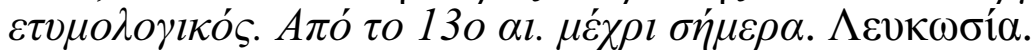

Germano, G. 1622: Vocabolario italiano et greco. Rom. 
Hansen, P. A. 2005: Hesychius. Lexicon. Vol. 3: П- - . Berlin; New York. Heldreich, T. von 1878: La faune de Grèce. Athen.

Kaibel, G. 1887: Athenaeus. Dipnosophistae. Vol. 1: libri 1-5. Leipzig.

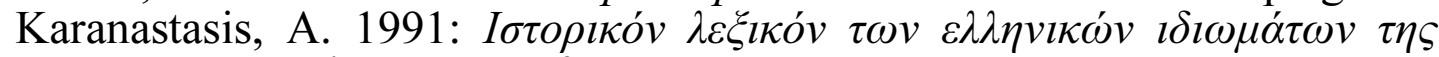

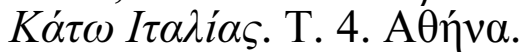

Karpov, V. 1996: Aristotel'. Istoriya zhivotnykh. Podgotovil k pechati Starostin, B. A. [Aristotle. History of animals. Prepared for publication by Starostin, B. A.]. Moscow.

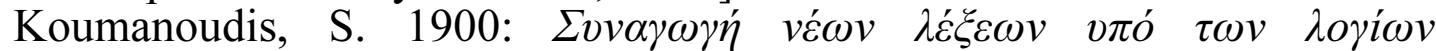

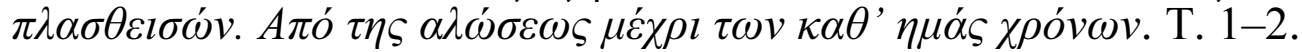
Athen.

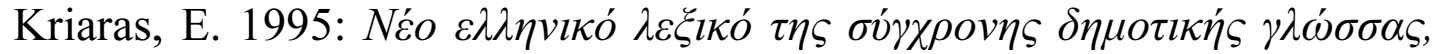

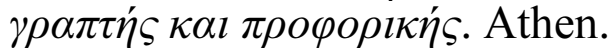

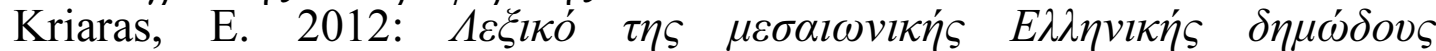

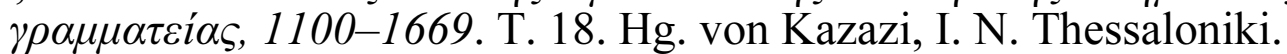

Lampe, G. W. H. 1961: A patristic Greek lexicon. Oxford.

Lanza, D., Vegetti, M., 1971: Aristotele. Opere biologiche. Torino.

Lavidas, N. 2018: Language change and early dictionaries of Modern Greek. In: Giannakis, G. K., u. a. (Hgg.). Studies in Greek Lexicography. Berlin; Boston.

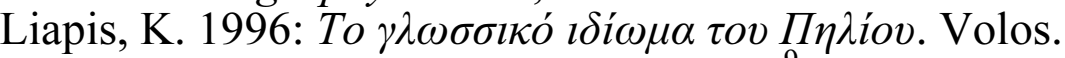

Liddell, H. G., Scott, R., Jones, H. S. ${ }^{9} 1940$ : A Greek-English lexicon. Oxford.

Louis, P. 1969: Aristote. Histoire des animaux. T. 3. Livres 8-10. Paris.

Mackridge, P. 2009: Language and national identity in Greece, 1766 1976. Oxford.

Manutius, A. 1497: Aristoteles. Opera. T. 3. Venedig (GW 2334).

Meursius, J. ${ }^{2}$ 1614: Glossarium Graeco-barbarum. Leiden (Die erste Edition von 1610 ist uns unzugänglich geblieben).

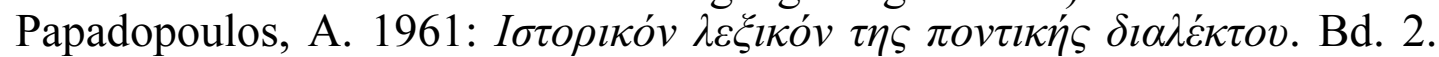
$\mathrm{M}-\Omega$. Athen.

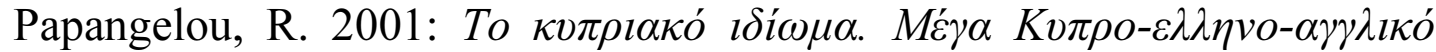

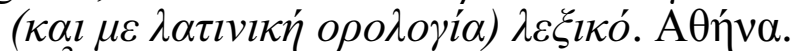

Pape, W. ${ }^{3}$ 1880: Handwörterbuch der Griechischen Sprache. 4 Bdd. Bd. 2. Griechisch-Deutsches Wörterbuch. 1- $\Omega$. Braunschweig.

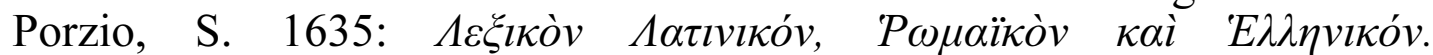
Dictionarium Latinum, Graeco-barbarum, et litterale. Paris.

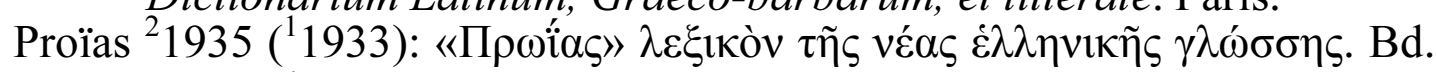
$1-2$. Athen.

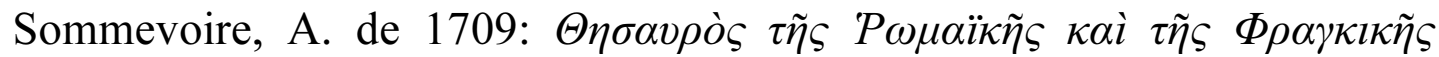
$\gamma \lambda \omega ́ \sigma \sigma \alpha \varsigma$. Tesoro della lingua greca-volgare ed italiana. Paris.

Sophocles, E. A. ${ }^{2} 1900\left({ }^{1} 1870\right)$ : Greek lexicon of the Roman and Byzantine periods (from B. C. 146 to A. D. 1100). New York.

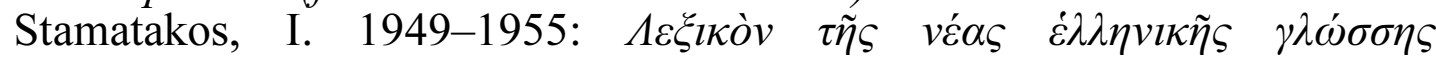

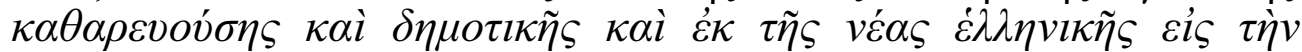
a $\rho \chi \alpha i \alpha v$. Bd. 1-2. Athen.

Stathi, E. 2006: Greek lexicography, modern. In: Brown, K. Encyclopedia of language and linguistics. 2nd edition. Vol. 5. Oxford. 147-149. 
Sundevall, C. J. 1863: Die Thierarten des Aristoteles von den Klassen der Säugethiere, Vögel, Reptilien und Insekten. Übersetzung aus dem Schwedischen. Stockholm.

Thompson, D. W. 1895: A glossary of Greek birds. Oxford.

Trapp, E., et al. 2001: Lexikon zur byzantinischen Gräzität. Wien (http://stephanus.tlg.uci.edu/lbg/\#eid=1\&context=lsj, letzter Zugriff am 8.2.2019).

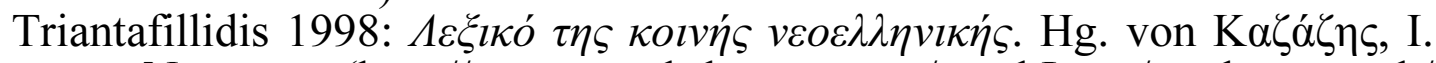
N., u. a. (http://www.greek-language.gr/greekLang/modern_greek/ tools/lexica/triantafyllides/index.html, letzter Zugriff am 8.2.2019)

Tricot, J. 1957: Aristote. Histoire des animaux. T. 2. Nouvelle traduction avec introduction, notes et index par Jean Tricot. Paris.

Turner, W. 1544: Turner W. Avium praecipuarum, quarum apud Plinium et Aristotelem mentio est, brevis et succincta historia. Köln.

Vinding, R. 1702: Eutecnii sophistae paraphrasis prosaica in Oppiani Ixeutica. Kopenhagen.

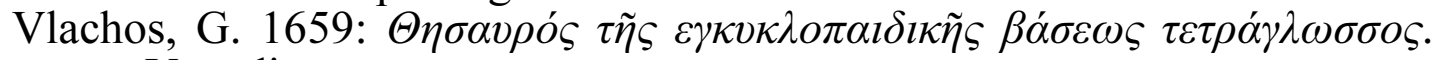
Venedig.

Vorobyev, G. 2018: Sylvia: Zur Entstehung des wissenschaftlichen Namens der Grasmücke (Arist. Hist. an. 592b22). Philologia Classica 13(2). 247-264.

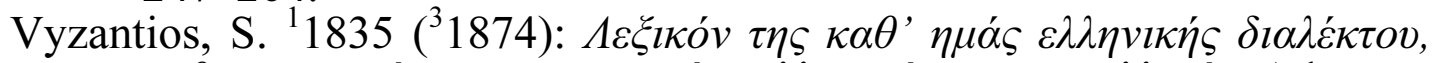

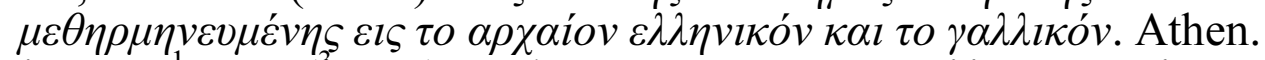

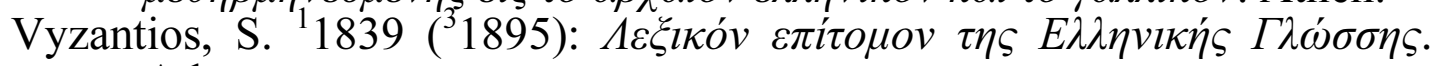
Athen.

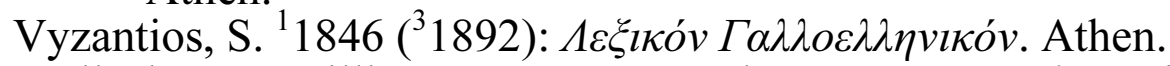

Wallach, V., Williams, K. L., Boundy J. 2014: Snakes of the world. A catalogue of living and extinct species. Boca Raton; London; New York. 